ance of such an instrument as the theodolite when carefully made is sufficient proof that axes designed as straight or slightly tapering cylindrical members fitting their mating elements with extensive surface contact are good enough for any instrument and nothing further need be said. But it is not possible without extraordinary precautions to make geometrically perfect cylindrical elements, and though the deviation from geometrically circular form in manufactured elements may not be sufficiently great to affect the readings of a theodolite by axis shift during rotation of telescope or circles, it may easily give rise to a serious source of error by slight but variable resistance to motion which is communicated to the instrument indications as a strain shift in an irregular manner. With decrease of the tolerances a more pronounced effect of this kind during relative motion of the two elements would be expected, and when the clearance between the functional surfaces reaches very small dimensions, the shearing of the lubricant may also cause the pointings and circle readings to change with time.

These defects will appear in the indications as systematic errors confused with the accidental errors, since the probable error is merely a measure of the agreement of average repeated readings and is not a measure of the accuracy of a reading. The two kinds of error can be separated, however, by devising special tests to establish a standard probable error of the accidental errors peculiar to the instruments and methods used. The systematic errors will then be revealed as an increment of the standard probable error.

It is by such specially arranged tests on the Wild instruments that Rannie and Dennis have been able to separate the systematic errors known to exist. "All of the evidence, . . . , indicated that angular errors of appreciable magnitude - of the order of $2^{\prime \prime}$ to $4^{\prime \prime}$ - may have resulted from strain or other axis trouble in at least nine of the 10 Wild theodolites examined."

The authors then proceeded to modify the alidade and telescope axes so that the unavoidable imperfections of geometric form would have no effect upon the instrument indications. That is to say, when the telescope was rotated in azimuth or transited, no irregular resistance to the movement would take place and consequently no elastic deformations would upset the circle readings.

The original paper should be consulted for the details of the modifications the authors were able to make, but it is sufficient to state here that the alterations to the axes conformed to kinematical principles as closely as the existing design would permit. After each instrument had been remodelled in this way, it was again taken through the previous tests, which showed that not only had the systematic errors been eliminated but also that in addition the accidental errors had been slightly reduced.

This paper, embodying the results of exhaustive tests upon the performance of certain theodolites by the Chief of the Triangulation Division and the District Engineer of the Geodetic Survey of Canada, surely must be regarded as one of the most important, if not the most important, contribution to the design of theodolites in particular and of measuring instruments in general which has hitherto been published.

It has conclusively shown that the usual machinetool design applied to an instrument of high precision, no matter how perfect the workmanship may be, is fundamentally wrong. It has shown that even an approach to correct or kinematic design of the couplings at once improves the reliability of the instrument indications, and incidentally it has shown that equally strict attention must be given to the design of subsidiary parts, such as levelling screws, if the best results are to be obtained.

$$
\text { A. F. C. Pollard. }
$$

Imperial College of Science and Technology, South Kensington, S.W.7.

\section{Aug. 3.}

1 "Improving the Performance of Primary Triangulation Theodolites as a result of Laboratory Tests", Canadian J. Research, 10, 347 ; 1934 .

\section{Accuracy of Least Squares Solutions}

I HAVE recently obtained the solution of this problem : Given $n$ linear equations of condition in $m$ unknowns, and that (1) the set of errors of the equations of condition are a sample drawn at random from a normal population of unknown standard deviation $\sigma$, and that (2) nothing more is known about the values of the unknowns or of $\sigma$ than that which can be inferred from the equations of condition, then $(A)$ what are the values of the unknowns? By rigorous, direct methods, and with no further assumptions, I have obtained the distribution functions in answer to question $(A)$. They are the same as those which Jeffreys ${ }^{1}$ obtained in semiintuitive fashion, by making the somewhat arbitrary assumption that the "prior probability" that $\sigma$ lies in a certain range $d \sigma$ is proportional simply to $d \sigma / \sigma$.

I have also obtained by direct arguments, with no assumptions other than (1) and (2), the answer to question $(B)$ : What is the value of $\sigma$ ? This distribution function is considerably more com plicated, involving $m$ and $\Sigma \delta^{2}$ as it should, than $d \sigma / \sigma$; and if one uses the function as a "prior probability", one can obtain in this second, indirect fashion the same answer as before to question $(A)$. I think that Jeffreys's conclusions were highly creditable to his insight, although I am compelled to agree with Fisher ${ }^{2}$ and Bartlett ${ }^{3}$ that his arguments were fallacious. I shall publish the new treatments in due course. T. E. STERNE.

Harvard College Observatory, Cambridge, Mass. June 25.

1 Jeffreys, Proc. Roy. Soc., A, 138, 48; 1932.

2 Fisher, Proc. Roy. Soc., A, 139, 343; 1933.

s Bartlett, Proc. Roy. Soc., A, 141, 518 ; 1933.

\section{Velocity of Reactions in Solution}

IN a recent publication, Williams and Hinshelwood ${ }^{1}$ reach the important conclusion that the influence of substituents on the velocity of benzoylation of aromatic amines in benzene solution depends principally on changes in the activation energy, that is, $E$ in the expression $k=P Z e^{-E / R T}$. Changes in $P$, a factor independent of the activation rate, are of much smaller importance as long as the medium is unchanged.

This publication is welcomed as affording lateral support to the conclusion already reached by my collaborators and myself on the basis of (1) the temperature effects, and (2) the additive effects observed, that the relative rates of chlorination of substituted phenyl and tolyl ethers in 99 per cent acetic acid are dependent solely on the activation energies. A summary and discussion of these results, which appear to have been overlooked by Williams 